\title{
DOES COMPUTERISED PERIMETRY OFFER PRACTICAL ADVANCES IN CHOICE OF THERAPY IN THE GLAUCOMA PATIENT?
}

\author{
H. DUNBAR HOSKINS JR \\ San Francisco
}

The title of this paper poses the question of whether computer assisted techniques in perimetry offer useful advantages in the management of the glaucoma patient. Answering this question requires an understanding of the advantages and limitations of computerised perimetry.

Effective computerised perimetry became available to a few institutions with the advent of the Octopus Perimeter. This was a large, expensive but flexible perimeter which could perform a variety of standardised static tests with minimal technician interaction. It could store the results of the tests and recall them for comparison to subsequent tests.

The key factor that ignited the interest of those involved in visual field examination was this ability to perform the same test over and over again in a standard manner. This eliminated one of the great variables that exists in manual perimetry. No matter how skilled the manual perimetrist, it is impossible for him/her to perform the identical test each time. The elimination of this variable offered the promise of standardised perimetry.

With the advent of the Humphrey Field Analyser (HFA), the cost of the instrumentation fell within the reach of most ophthalmology centres and the era of standardised visual field testing began. We are approaching a decade of experience with these computerised techniques and it seems reasonable to examine whether they have truly brought advantage to the patient and the physician.

\section{THE PURPOSE OF VISUAL FIELD TESTING IN GLAUCOMA ${ }^{1,2}$}

In the patient who has or is suspected of having glaucoma, we are interested in answering one of two questions:

(1) Is there visual field damage?

(2) Has the visual damage changed?

In order to know if damage exists we must first be able to define the absence of damage. Visual field examination

Supported by the Foundation for Glaucoma Research, San Francisco, California.

Correspondence to: H. Dunbar Hoskins, Jr MD, Clinical Professor of Ophthalmology, University of California, San Francisco Medical Center, San Franscisco, California. by current techniques requires the patient to perceive a brighter stimulus presented on a dimmer background. This so-called differential light sensitivity is the basis of the field test. The differential light sensitivity is influenced by many factors including age, refractive correction, media clarity, patient attentiveness and responsiveness and perhaps other systemic factors of which we are not aware. Usually we are not trying to measure these factors when we perform a visual field examination. More likely we are trying to measure some degree of retinal, optic nerve or visual pathway pathology.

In order to separate pathologic change from normal ageing change or from changes in media clarity or from variations in patient performance, we are required to have standards and ways of interpreting variations from those standards.

With the advent of computerised perimetry and the standardisation of the test technique, investigators were able to test large numbers of 'normal' patients of varying ages with clear media to develop statistical definitions of the 'normal' response to the standardised test. Investigators determined that there was a deterioration in differential light sensitivity with age and that this deterioration was predictable, measurable and fell within certain limits. Thus, the normal state (the absence of damage) for each age group was defined and became available for comparison to the individual patient.

This might have been done for manual perimetry but, unfortunately, the results would have been valid only for the technician who did it and only as long as that technician did the test the same way each time. The beauty of computerised perimetry is that the standards developed for the Humphrey field analyser are applicable to all Humphrey field analysers and the same is true for the Octopus perimeter.

By having normal standards, we are better able to recognise deviations from these standards in patients with early diffuse visual field loss that might otherwise be considered normal. This may even occur when both eyes fall within the statistical range of 'normal' but one eye has a

Eye (1992) 6, 43-46 


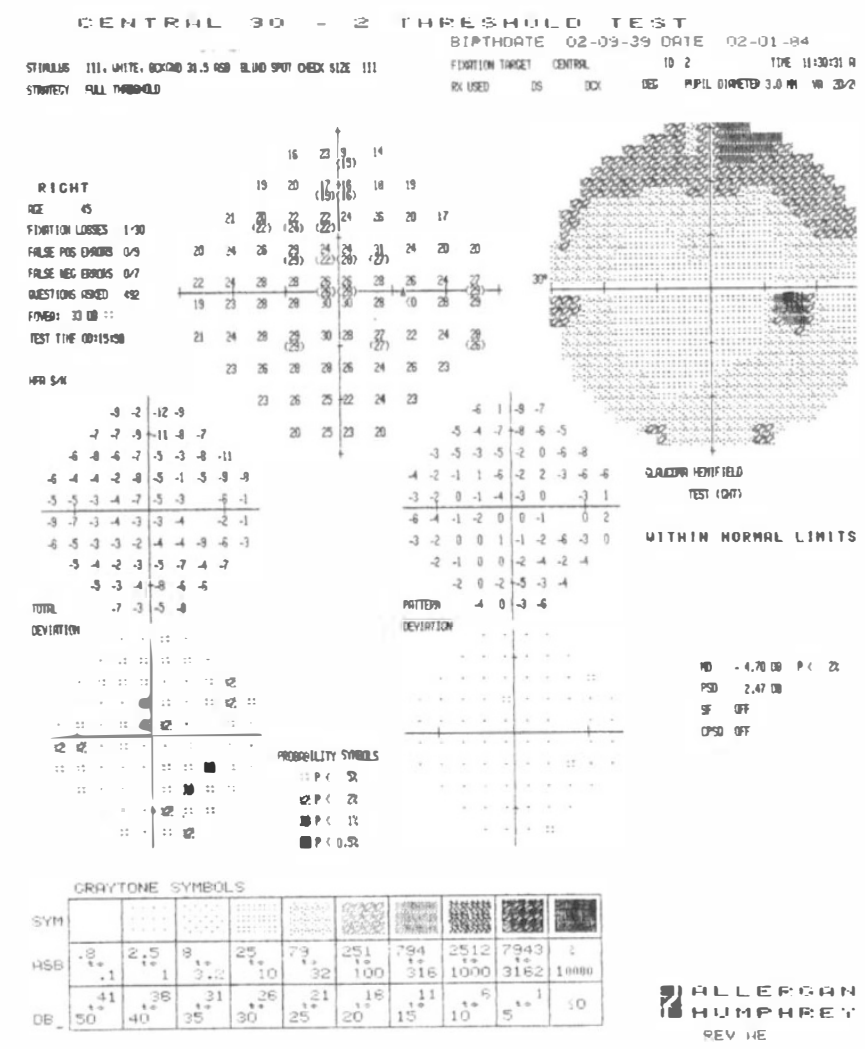

Fig. 1 Graphic Printout of Visual Field Test. The Gray scale (upper right) seems to indicate depression in the upper part of the field. The test results are compared to the normal data for $a$ patient of this age and the difference depicted in the Total Deviation plot (lower left). This indicates that there is generalised loss. The Pattern Deviation Plot shows no particular localised loss. This patient had a cataract without glaucoma.

larger cup/disc ratio, a higher pressure a lower mean sensitivity value on visual field testing. In my opinion, such patients can be categorised as having glaucoma and deserve a trial of therapy.

The ability to test large numbers of patients in a standard manner also gave us the ability to measure how differential light sensitivity varies in different portions of the eye. We know, for example that the sensitivity is greatest around fixation and falls off toward the periphery. We also know that the rate of decline toward the periphery is greater in the upper half of the field than the lower half., By using these measurements we can compare different portions of the field to other portions in the same eye to determine if a particular area is functioning below expectations even when the function of the eye as a whole may be within normal limits.

This testing for localised change occurs in the Humphrey Statpak II Glaucoma Hemifield Test (GHT) and may allow for earlier recognition of glaucomatous damage.

\section{LOCAL VS DIFFUSE DAMAGE}

Diffuse damage to visual function may be the earliest sign of glaucoma but it is also seen with ageing and with cataract development. Computerised perimetry not only provides us with standards for age but also provides a technique which helps sort out glaucomatous visual loss form that due to progression for cataract. This is the Pattern Deviation Plot.

Computers have an amazing facility to manipulate numeric data and provide graphic output. The human brain is quite poor by comparison in manipulating numeric data but it excels at interpreting patterns. Thus computerised perimetry utilises the power of the computer to manipulate numbers and then presents the information in such a way as to use the power of the brain for interpretation.

The Total Deviation and Pattern Deviation plots provided on the Humphrey printout are typical of this (Figs. 1-3). The total Deviation Plot depicts all those points in the visual field that deviate from an expected normal response. It depicts them in such a way as to indicate the magnitude of the deviation.

The Pattern Deviation Plot uses the same raw patient data but manipulates it in such a way as to remove any diffuse loss from the graphic presentaton. It then presents those areas that are worse than the diffuse loss and are more likely to be localised areas of damage. This can be very helpful in recognising localised glaucomatous damage in a patient with early cataract change or in following a patient with glaucoma who has a worsening cataract.

\section{RECOGNISING CHANGE}

The second major requirement of perimetry is to recognise change in a previously defined visual field defect. This is a considerably more difficult problem because of fluctuation.

In sequential visual fields performed on the eye, change

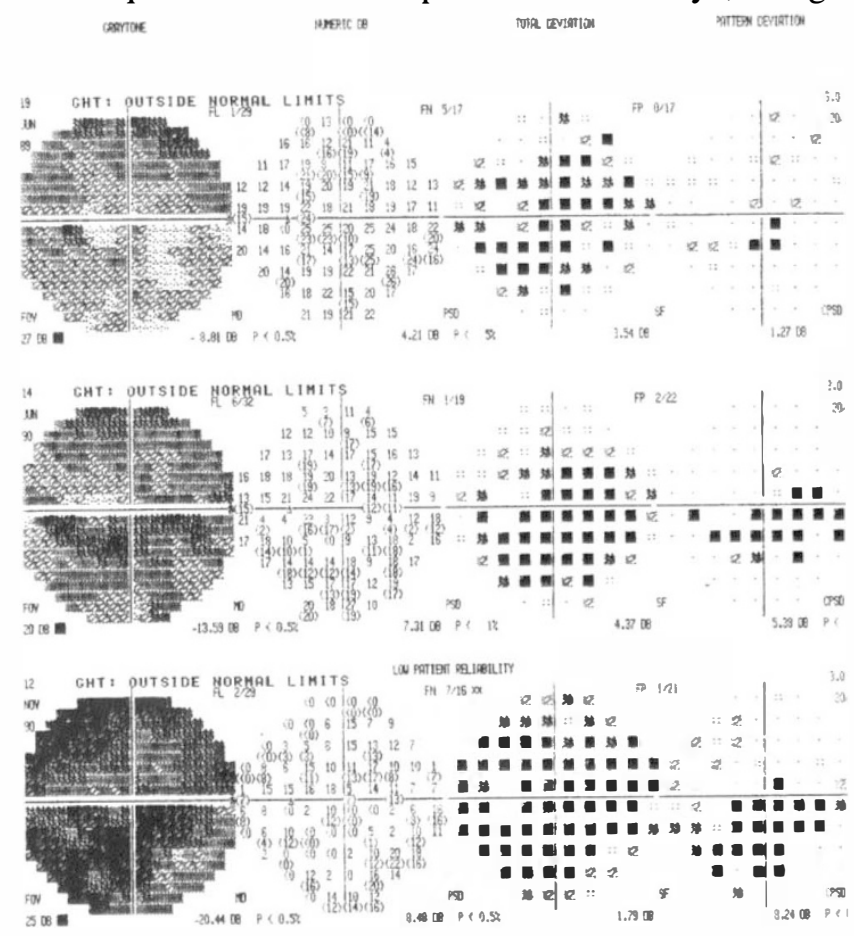

Fig. 2 Glaucomatous Progression. This elderly patient refused surgery. He had both cataract and glaucoma. The Graytones, Total Deviation Plots and the Pattern Deviation Plots all are worsening, indicating progression of both diseases. 


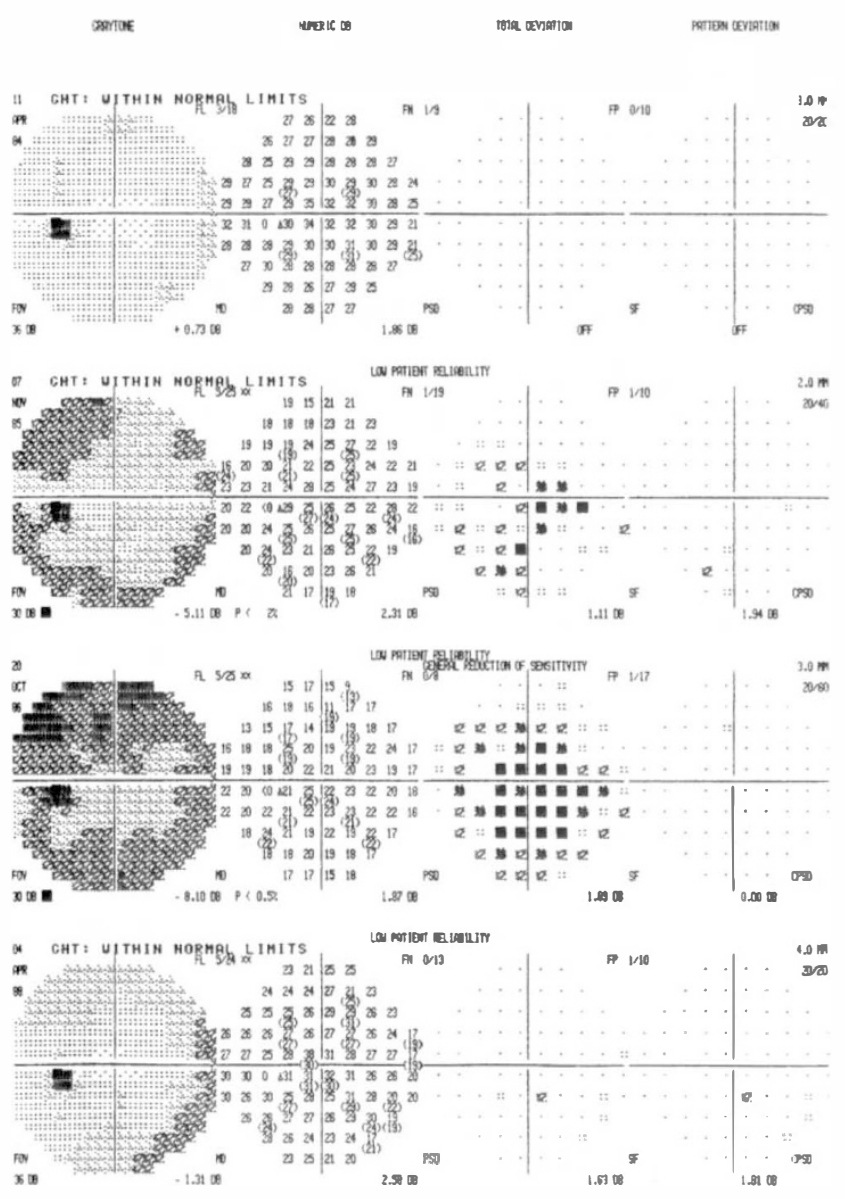

Fig. 3 Cataract Progression. Note worsening of Graytone and Total Deviation Plots which improve in the last examination. Cataract surgery was done betw'een the third and fourth fields. The continued normal appearance of the Pattern Deviation Plot helps differentiate this from glaucomatous damage.

will occur. The totality of this change includes that due to artefact, that due to pathology and that due to fluctuation. Since we are only interested in the change due to pathology we must make every effort to eliminate or measure change due to artefact or fluctuation.

There are numerous sources of artefactual change in visual field testing. ${ }^{5}$ In order to minimise artefactual changes, wherever possible these sources of artefacts should be controlled by making them as much the same as possible as the previous test. For example, pupil size should not vary from test to test. The best corrected vision with the appropriate near add should be provided for each test, etc.

If artefacts are minimised, fluctuation can be usefully defined as the variation in visual field results which is not due to change in pathology. It is not quite as simple as that because it is impossible to separate fluctuation from pathology. ${ }^{6.7}$ Indeed fluctuation increases with pathology. Therefore we must measure fluctuation and define it in relation to pathologic change.

In the statistical interpretation of visual fields, fluctuation can be precisely defined and measured. ${ }^{9}$ Short-term fluctuation is that which occurs within a given test. In the HFA ten locations are measured twice and the amount that the second test for each location varies from the first is used in calculating the short-term fluctuation. This is normally between 1.5 and $2.5 \mathrm{Db}$. Long-term fluctuation is that which occurs between two tests in an otherwise unchanging eye. Generally it is around $1 \mathrm{Db}$ in normal patients but may be greater if extensive damage exists.

If we eliminate change due to artefact and change due to fluctuation, we are left with change due to pathology. This is what we want to measure. The computer assists us in this by performing linear regression analysis on a series of fields, analysing the entire field and the individual spots. It presents the results of this analysis as a probability that the change is outside the normal variation expected. ${ }^{10.11}$

It is this computerised analysis that we all seek, to help us determine whether a patient has truly worsened. Computerised perimetry offers us real advantage over manual perimetry in accomplishing this. In order to receive the greatest advantage from these statistical techniques, it is necessary to have sufficient numbers of observations.

At least five and preferably more visual field tests are necessary to perform accurate linear regression. Thus the trend is to perform fields more frequently in order to have enough data points for analysis. One approach is to perform three fields within the first month to provide a baseline. If a subsequent field appears changed then it can be repeated to provide two subsequent fields to compare to the three original ones.

The Humphrey Statpac II program" averages two initial fields and compares each subsequent field to that average. It gives the calculated change in mean defect and the statistical probability that it is within normal limits. It also performs a similar analysis for each location tested in the field and identifies those that have worsened or improved at the $95 \%$ confidence level. As one follows these individual points it is obvious that there is a great deal of variation in point change in patients who are apparently stable. Therefore, unless there is consistent or clustered change, it is unwise to put much emphasis on change of one or two spots.

\section{SUMMARY OF COMPUTERISED PERIMETRY}

Computerised perimetry has several distinct advantages over manual perimetry in managing glaucoma patients. Most important of these is the standardisation of the test which allows comparison of fields done by different technicians and even at different centres. This also allowed the development of age related normal standards based on examination of large numbers of normal patients of all ages. Another great advantage is that computerised perimetry demands less from the technician. An untrained individual can be performing excellent visual field tests after only 30 minutes instruction. This can effectively reduce the cost of each field performed.

The analysis techniques provided by computerised perimetry have revealed the large amount of 'noise' that exists in measuring almost any biologic system. This 'noise', which we call fluctuation, complicates the interpretation of results. One has two choices; either wait until 
the change is so great as to be certain that it is not due to the noise alone or use statistical techniques to recognise change earlier. This latter requires computerised perimetry and more data points thus increasing the burden on the patient.

The goal of future research is further to refine the testing technique to reduce wasted effort and enhance the analysis techniques. Because glaucoma is a slowly progressive disease this process is relatively slow. It involves collecting the data on patients who have demonstrated change and analysing that data to attempt recognition of patterns which would allow earlier diagnosis of change. It is a challenging process but promises further assistance in managing our patients.

The author is a technical consultant to Allergan-Humphrey.

\section{REFERENCES}

1. Hoskins HD and Kass M: Becker-Shaffer's Diagnosis and Therapy of the Glaucomas, Sixth Edition, 118-170, 1989, The CV Mosby Co.
2. Anderson, DR: Testing the field of vision, St Louis, 1982, The CV Mosby Co.

3. Hoskins HD Jr and Migliazzo C: Development of a visual field screening test using a Humphrey visual field analyser, Doc Ophthalmol Proc Series 1985, 42: 85-90.

4. Katz J and Sommer A: Asymmetry and variation in the normal hill of vision, Arch Ophthalmol 1986, 104: 65

5. Hoskins HD and Kass M: Becker-Shaffer's Diagnosis and Therapy of the Glaucomas, Sixth Edition 126-189, 1989, The CV Mosby Co.

6. Flammer J, Drance SM, Fankhauser F, Augustiny L: Differential light threshold in automated static perimetry: factors influencing short-term fluctuation, Arch Ophthalmol 1984, 102: 876.

7. Magee SD, Hoskins HD, Kidd MN: Long-term fluctuation in glaucomatous visual fields: a point by point analysis. Paper presented at the annual meeting of the Association for Research in Vision and Ophthalmology, May 1987.

8. Flammer J, Drance SM, Augustiny L, Funkhouser A: Quantification of glaucomatous visual field defects with automated perimetry, Invest Ophthalmol Vis Sci 1985, 26: 176.

9. Allergan Humphrey: Statpac User's Guide, 1989.

10. Allergan Humphrey: Introducing Statpac 2, New Releases for the Humphrey Field Analyser, 1989. 